\title{
Bevacizumab is more effective in nasopharyngeal carcinoma patients with lower maximum radiation dose to the temporal lobe
}

\author{
Adam Garsa ${ }^{1}$, Jennifer C. Ho ${ }^{1}$, Chaosu $\mathrm{Hu}^{2}$, Eric L. Chang ${ }^{1}$ \\ ${ }^{1}$ Department of Radiation Oncology, Norris Cancer Hospital, Keck School of Medicine of USC, Los Angeles, CA, USA; ${ }^{2}$ Department of Radiation \\ Oncology, Fudan University Shanghai Cancer Center, Shanghai 200032, China \\ Correspondence to: Eric L. Chang, MD, FASTRO. Norris Cancer Hospital, Keck School of Medicine of USC, 1441 Eastlake Ave, NOR-G356, Los \\ Angeles, CA 90033, USA. Email: eric.chang@med.usc.edu. \\ Comment on: Li Y, Huang X, Jiang J, et al. Clinical Variables for Prediction of the Therapeutic Effects of Bevacizumab Monotherapy in \\ Nasopharyngeal Carcinoma Patients With Radiation-Induced Brain Necrosis. Int J Radiat Oncol Biol Phys 2018;100:621-9.
}

Submitted Feb 23, 2019. Accepted for publication Feb 26, 2019.

doi: $10.21037 /$ cco.2019.02.06

View this article at: http://dx.doi.org/10.21037/cco.2019.02.06

Nasopharyngeal carcinoma (NPC), due to its location and routes of spread, requires radiation therapy to the skull base and may include a portion of the temporal lobes of the brain in the high dose volume by virtue of its anatomic location. Radiation-induced brain necrosis ( $\mathrm{RN}$ ) usually develops months to years after radiation therapy. It is histologically characterized by fibrinoid changes to the blood vessels and ischemia leading to coagulative necrosis of the brain parenchyma and demyelination $(1,2)$. On imaging, radiation treatment-related changes in the temporal lobes can sometimes be difficult to differentiate from intracranial tumor recurrence. After radiation therapy, magnetic resonance imaging (MRI) may commonly show mild cerebral edema or white matter changes (3). $\mathrm{RN}$ is a severe form of late radiation toxicity to the central nervous system. It causes necrotizing leukoencephalopathy, which appears as confluent regions of T2/FLAIR hyperintensity on MRI, often associated with edema and heterogenous enhancement. Additional imaging techniques and sequences including MR perfusion, MR spectroscopy, susceptibilityweighted imaging (SWI), diffusion-weighted imaging (DWI) and positron emission tomography (PET) can be useful to establish the diagnosis $(4,5)$.

Radiation treatment-related changes noted on imaging may be asymptomatic or symptomatic. If changes are asymptomatic and mild, conservative follow-up is appropriate, as these radiographic changes will often improve or stabilize with time (3). Symptoms of RN may include cognitive dysfunction, memory impairment, dizziness, seizures, headaches, personality changes or confusion (6). Management options for symptomatic RN may include corticosteroids, bevacizumab or surgery.

In this article, $\mathrm{Li}$ et al. performed a retrospective review of 50 patients who were treated with bevacizumab for $\mathrm{RN}$ that developed after receiving radiation therapy for nasopharyngeal cancer (7). All patients were followed for 6 months, and clinical data as well as brain edema volume based on MRI findings were analyzed. Patients were included if they completed radiation therapy at least 6 months prior, had no prior treatment of $\mathrm{RN}$ with corticosteroids, and no evidence of tumor recurrence or metastases. They were treated with bevacizumab $5 \mathrm{mg} / \mathrm{kg}$ intravenously once every 2 weeks for up to 4 courses.

Seventy-six percent of patients had an effective response, defined as a $\geq 25 \%$ reduction in brain edema volume, with a median decrease in RN volume seen on T2/FLAIR of $73 \%$. The maximum dose to the temporal lobe $\left(\mathrm{D}_{\max }\right)$ and total radiation dose of the neck were the only two factors that were significantly different between patients with and without an effective response. A random forest model analysis identified $\mathrm{D}_{\max }$ of the temporal lobe as the main predictor of effective response with a threshold of 75.5 Gy, with an accuracy rate of $94.7 \%$ for predicting effectiveness of bevacizumab. These results suggest that bevacizumab is more effective in reducing brain edema in patients with temporal lobe $\mathrm{D}_{\max }$ less than $75.5 \mathrm{~Gy}$.

Fifteen out of 38 patients had recurrence of $\mathrm{RN}$, and shorter duration between radiation therapy and either $\mathrm{RN}$ 
diagnosis or bevacizumab treatment were found to be the top two predictors of $\mathrm{RN}$ recurrence. The authors also analyzed baseline serum VEGF levels which were only available in 15 patients, but found no significant difference between patients with and without an effective response. Limitations of the study include lack of details about how patients were diagnosed with $\mathrm{RN}$, whether they had to be symptomatic to be included, and whether patients received any additional treatment with corticosteroids, and had longer follow-up.

Nevertheless, this article by Li et al. contributes significantly to the literature on treatment of $\mathrm{RN}$ with bevacizumab (7). In general, asymptomatic $\mathrm{RN}$ can be managed conservatively without intervention, and in patients who are symptomatic from cerebral edema, corticosteroids can be used as a first-line therapy. The data for bevacizumab appears promising but is based on small retrospective studies and two prospective randomized trials. A double-blind clinical trial from MD Anderson randomized patients with $\mathrm{RN}$ (with progressive neurologic signs or symptoms) from treatment for various head and neck and central nervous system tumors, to receive either bevacizumab $(7.5 \mathrm{mg} / \mathrm{kg}$ once every 3 weeks, up to 4 times) or placebo (8). Patients were allowed to receive dexamethasone. All bevacizumab treated patients $(5 / 5$ of the randomized patients and $7 / 7$ patients who crossed over) responded, while no placebo patients $(0 / 7)$ did. The median decrease in edema and in contrast enhancement was 59\% and $63 \%$, respectively, in those treated with bevacizumab. Another open label trial was published last year by the same group as the current study by $\mathrm{Li}$ et al., which randomized 112 patients with RN from radiation therapy for NPC to receive either bevacizumab $(5 \mathrm{mg} / \mathrm{kg}$ every 2 weeks, for up to 4 courses) or steroids (methylprednisolone $500 \mathrm{mg}$ IV daily for 3 days, followed by oral prednisone for 2 months) (9). A greater percentage of patients randomly assigned to the bevacizumab arm had a radiographic response compared to patients randomly assigned to the steroid arm $(65.5 \%$ versus $31.5 \%, \mathrm{P}<0.001)$, with a corresponding decrease in edema volume of $52 \%$ versus $19 \%$, and reduction in enhancement of $25 \%$ versus $5 \%$. Bevacizumab remained significant on multivariate analysis for treatment outcome. The bevacizumab group also had greater proportional benefit in terms of improvement in neurological signs/symptoms and cognitive function. There was no difference in the rate of $\mathrm{RN}$ recurrence, with 12 and 11 patients developing $\mathrm{RN}$ recurrence in the bevacizumab and steroid arms, respectively.
In addition to these prospective trials, there have been a few retrospective series, including the current study which to our knowledge represents the largest retrospective experience reported in the literature. In a recent metaanalysis of bevacizumab for the treatment of RN by Delishaj et al., approximately $91 \%$ of patients had a clinical benefit after bevacizumab, 98\% had radiographic improvement, and of the studies that analyzed pre- and post-treatment imaging, there was a median decrease in T1 contrast enhancement and T2/FLAIR signal abnormality of $64 \%$ and $60 \%$, respectively (10).

The risk of developing RN after radiation for NPC is dependent on several treatment-related factors. In a large retrospective review of 849 patients with NPC treated with radiotherapy alone, Yeh et al. reported a higher incidence of $\mathrm{RN}$ with doses above $72 \mathrm{~Gy}(11 \%)$ versus less than $72 \mathrm{~Gy}$ (4\%) (11). Lee et al. reviewed 1,032 patients with early-stage NPC and found that higher dose per fraction and B.I.D. fractionation were independent risk factors for symptomatic necrosis (12).

Intensity-modulated radiation therapy (IMRT) can reduce the radiation dose and volume to the temporal lobes compared with $2 \mathrm{D}$ or $3 \mathrm{D}$ conformal techniques (13). In a prospective, randomized trial comparing IMRT with 2D conventional radiation, the rate of temporal lobe necrosis was significantly lower in the IMRT group (13\% vs. $21 \%$ ) (14). The use of induction chemotherapy or adaptive radiation planning may allow for additional dose reduction to the temporal lobes in patients with locally advanced disease (15).

Treatment with bevacizumab carries significant cost and a risk of adverse events, so there is a need to identify factors associated with a favorable response. In the present study by Li et al., the inclusion criteria required radiographic evidence of $\mathrm{RN}$ but did not require patients to be symptomatic. It is unclear how many asymptomatic patients were included in this study, but we believe that asymptomatic patients can be observed. Patients with $\mathrm{D}_{\max }$ to the temporal lobes of $>75.5$ Gy had lower rates of response to bevacizumab, and a short interval between radiation therapy and $\mathrm{RN}$ was associated with a higher risk of $\mathrm{RN}$ recurrence. It is unclear if these factors correlate with a more irreversible $\mathrm{RN}$ process that is refractory to medical management, or if bevacizumab in particular is less effective in these settings.

This study by $\mathrm{Li}$ et al. raises other interesting questions: what is the optimal bevacizumab dosing and number of cycles to be given for RN? Several different bevacizumab 
dosages have been reported in the literature, but it is unclear which one is optimum. Is there an additional benefit with concurrent or sequential corticosteroid treatment? Bevacizumab remains a promising treatment for $\mathrm{RN}$, with high overall rates of radiographic and clinical response. It is our hope that future studies will help further refine management of brain $\mathrm{RN}$ in the setting of follow-up care after definitive treatment of NPC.

\section{Acknowledgments}

None.

\section{Footnote}

Conflicts of Interest: The authors have no conflicts of interest to declare.

\section{References}

1. Chen J, Dassarath M, Yin Z, et al. Radiation induced temporal lobe necrosis in patients with nasopharyngeal carcinoma: a review of new avenues in its management. Radiat Oncol 2011;6:128.

2. Giglio P, Gilbert MR. Cerebral radiation necrosis. Neurologist 2003;9:180-8.

3. Walker AJ, Ruzevick J, Malayeri AA, et al. Postradiation imaging changes in the CNS: how can we differentiate between treatment effect and disease progression? Future Oncol 2014;10:1277-97.

4. Chao ST, Ahluwalia MS, Barnett GH, et al. Challenges with the diagnosis and treatment of cerebral radiation necrosis. Int J Radiat Oncol Biol Phys 2013;87:449-57.

5. Landry D, Garsa AA, Glastonbury CM. Imaging of cerebral radionecrosis: collateral damage from head and neck radiation. Neurographics 2016;6:151-8.

6. Lee $\mathrm{AW}, \mathrm{Ng} \mathrm{SH}, \mathrm{Ho} \mathrm{JH}$, et al. Clinical diagnosis of late temporal lobe necrosis following radiation therapy for nasopharyngeal carcinoma. Cancer 1988;61:1535-42.

7. Li Y, Huang X, Jiang J, et al. Clinical Variables for Prediction of the Therapeutic Effects of Bevacizumab

Cite this article as: Garsa A, Ho JC, Hu C, Chang EL. Bevacizumab is more effective in nasopharyngeal carcinoma patients with lower maximum radiation dose to the temporal lobe. Chin Clin Oncol 2019;8(Suppl 1):S20. doi: 10.21037/ cco.2019.02.06
Monotherapy in Nasopharyngeal Carcinoma Patients With Radiation-Induced Brain Necrosis. Int J Radiat Oncol Biol Phys 2018;100:621-9.

8. Levin VA, Bidaut L, Hou P, et al. Randomized doubleblind placebo-controlled trial of bevacizumab therapy for radiation necrosis of the central nervous system. Int J Radiat Oncol Biol Phys 2011;79:1487-95.

9. Xu Y, Rong X, Hu W, et al. Bevacizumab Monotherapy Reduces Radiation-induced Brain Necrosis in Nasopharyngeal Carcinoma Patients: A Randomized Controlled Trial. Int J Radiat Oncol Biol Phys 2018;101:1087-95.

10. Delishaj D, Ursino S, Pasqualetti F, et al. Bevacizumab for the Treatment of Radiation-Induced Cerebral Necrosis: A Systematic Review of the Literature. J Clin Med Res 2017;9:273-80.

11. Yeh SA, Tang Y, Lui CC, et al. Treatment outcomes and late complications of 849 patients with nasopharyngeal carcinoma treated with radiotherapy alone. Int J Radiat Oncol Biol Phys 2005;62:672-9.

12. Lee AW, Kwong DL, Leung SF, et al. Factors affecting risk of symptomatic temporal lobe necrosis: significance of fractional dose and treatment time. Int J Radiat Oncol Biol Phys 2002;53:75-85.

13. Kam MK, Chau RM, Suen J, et al. Intensity-modulated radiotherapy in nasopharyngeal carcinoma: dosimetric advantage over conventional plans and feasibility of dose escalation. Int J Radiat Oncol Biol Phys 2003;56:145-57.

14. Peng G, Wang T, Yang KY, et al. A prospective, randomized study comparing outcomes and toxicities of intensity-modulated radiotherapy vs. conventional two-dimensional radiotherapy for the treatment of nasopharyngeal carcinoma. Radiother Oncol 2012;104:286-93.

15. Yang H, Chen X, Lin S, et al. Treatment outcomes after reduction of the target volume of intensity-modulated radiotherapy following induction chemotherapy in patients with locoregionally advanced nasopharyngeal carcinoma: A prospective, multi-center, randomized clinical trial. Radiother Oncol 2018;126:37-42. 